\title{
COLABORACIÓN DE LOS OBJETOS DE APRENDIZAJE EN LA GESTIÓN DEL APRENDIZAJE
}

\author{
(COLLABORATION OF LEARNING OBJECTS IN THE MANAGEMENT LEARNING)
}

\author{
Ma. de Lourdes Y. Margain Fuentes \\ Universidad Politécnica de Aguascalientes (México) \\ Francisco Álvarez Rodríguez \\ Jaime Muñoz Arteaga \\ Universidad Autónoma de Aguascalientes, (México)
}

\section{RESUMEN}

Este artículo presenta el avance de un proyecto de investigación cuyo objetivo es diseñar y evaluar una metodología basada en ingeniería de software instruccional para la gestión del aprendizaje. La propuesta del proyecto se concentra en la parte dinámica de la gestión del proceso enseñanza-aprendizaje, a través de la generación de mapas conceptuales en combinación con objetos de aprendizaje con la finalidad de interpretar el significado de los estudiantes y provocar la mejora continua.

Específicamente este artículo profundiza sobre la importancia del aspecto de la colaboración de Los objetos de aprendizaje. Para el LO se identifican tres fases que son fundamentales y que muestran el "know-how" de la colaboración de los objetos de aprendizaje en la gestión del aprendizaje.

\begin{abstract}
This work presents a research project advance, the target is design and evaluate a methodology based in instructional software engineering for learning management. The project's proposed focus in the dynamic part of learning process management by the generation of conceptual maps and learning objects, the intention is interpret the student meaning and produce the continuous improvement.

Specifically, this paper deepens about the value of learning objects collaborations issue. For that, we identified tree phases that are fundamental and show the "know-how" of learning objects collaboration in the learning management.
\end{abstract}


Ma. De Lourdes Margain, Francisco Álvarez y Jaime MuÑoz

COLABORACIÓN DE LOS OBJETOS DE APRENDIZAJE EN LA GESTIÓN DEL APRENDIZAJE

\section{INTRODUCCIÓN}

En la nueva era de la información la difusión masiva del uso de la tecnología de información y las comunicaciones ha transformado los entornos sociales en los que se encuentra inmerso el ser humano. Las tecnologías de información han transformado diversas industrias, han hecho surgir nuevas y han desaparecido otras.

En la industria de la educación la demanda por "aprender" en las instituciones en los últimos años ha aumentado considerablemente. Aguilar (2003). Y no sólo la demanda es hacia los medios tradicionales de educación, ahora con el avance de la ciencia y la tecnología, se percibe un factor que está provocando una revolución en el mercado hacia los diferentes sistemas de aprendizaje.

Las instituciones educativas que hoy en día se interesan en mejorar su sistema orientado al logro de la calidad de la educación, debe evolucionar acorde a la nueva era de la información y ser parte de los agentes de cambios tecnológicos que den origen a los nuevos procesos de gestión basados en tecnologías de información (TI).

Por gestión educativa se comprende al conjunto de actividades encaminadas a facilitar la transformación de las condiciones institucionales con espíritu de renovación, controversia y de investigación. En materia de TI para la gestión educativa surgen sistemas de gestión de aprendizaje, también conocidos como LMS (Learning Management Systems - por sus siglas en inglés).

Un sistema de gestión de aprendizaje, Zapata (2003) lo define como una herramienta informática y telemática organizada en función de unos objetivos formativos de forma integral [es decir que se puedan conseguir exclusivamente dentro de ella] y de unos principios de intervención psicopedagógica y organizativos.

En este sentido, las tecnologías en materia de aprendizaje en línea y en gestión de aprendizaje hoy en día convergen en metodologías, técnicas y uso de herramientas con enfoques cada vez más modernos

Nuestro interés y enfoque de investigación, consiste en acelerar esa convergencia en la gestión del aprendizaje, a través de la incorporación de los nuevos elementos digitales de computadora conocidos como objetos de aprendizaje -LO (Learning Objects) por sus siglas en inglés- y con sus características esenciales de uso y re-uso entender el rol que juegan desde el punto de vista del aprendizaje colaborativo. 
Ma. DE Lourdes Margain, Francisco Álvarez y Jaime MuñoZ

COLABORACIÓN DE LOS OBJETOS DE APRENDIZAJE EN LA GESTIÓN DEL APRENDIZAJE

\section{Aprendizaje colaborativo}

El aprendizaje colaborativo como lo definen en sus inicios Ausubel, Novak \& Hanesian (1978) es una actividad en la cual los estudiantes, y posiblemente sus maestros, construyen cooperativamente un modelo explícito de conocimiento.

En la literatura revisada, los modelos clásicos del aprendizaje colaborativo (Vega, G., Rojo, B., 1999), señalan varias técnicas o formas de aplicar el aprendizaje colaborativo, estas van desde fomentar el aprendizaje por experiencia, por descubrimiento, plantear de manera clara el establecimiento de metas y premios grupales, fomentar debates, explicaciones, ejercicios, etc., hasta hacer capaz al alumno de tomar responsabilidades en la gestión del grupo.

Algunos otros autores como Vizcaíno, A., Olivas, J., y Prieto, M. (2001) señalan como ventajas de los entornos de aprendizaje colaborativos el aumento del aprendizaje debido a la interacción entre personas dado que existe una mayor motivación para aprender y por el contrario remarcan que los procesos de aprendizaje que realiza un alumno cuando trabaja en solitario son diferentes de los que realiza cuando trabaja en grupo, por este motivo los modelos del estudiante hasta ahora existentes no son válidos para sistemas colaborativos.

Dada la incorporación y ventajas de los objetos de aprendizaje, el aspecto de la colaboración se retoma y se define en este trabajo como cualquier actividad que se realiza entre una o más entidades, las cuales pueden ser estudiantes, maestros $\mathrm{u}$ objetos.

\section{Objetos de aprendizaje}

Los Objetos de Aprendizaje, como hoy los conocemos fueron identificados en su concepción, como una manera de representar el conocimiento David Merrill (1990), en su fundamentación de la primera y segunda generación del diseño instruccional, propone -para la adquisición y representación del contenido de un curso- que el conocimiento debe ser representado en términos de objetos a los cuales les denomina "frames". Estos frames son clasificados en tipos, como entidades que responden a objetos, personas, criaturas, lugares o símbolos, actividades y procesos.

Mas tarde, la teoría base de $L O$ originada por David Wiley (2000), los fundamenta como un tipo de elemento nuevo de computadora y basado en el paradigma orientado 
Ma. De Lourdes Margain, Francisco Álvarez y Jaime MuÑoz

COLABORACIÓN DE LOS OBJETOS DE APRENDIZAJE EN LA GESTIÓN DEL APRENDIZAJE

a objetos en las ciencias de la computación. La idea principal del "objeto", es que puede ser "reutilizado". Además los define como una entidad, digital o no digital, la cual puede ser usada o rehusada o referenciada durante la tecnología instruccional para el aprendizaje en línea.

Tecnológicamente, los $L O$ son un nuevo tipo de medio electrónico digitalizado que apoya al proceso enseñanza-aprendizaje. Estos elementos digitales, no sólo son herramientas de apoyo para la educación a distancia, también lo son para la educación presencial e incluso algunos autores señalan y dan importancia a que sean tratados para la educación personalizada e individualizada (Bermejo, Treviño 2002). A la par los objetos de aprendizaje son utilizados también para programas de capacitación en web como se presenta en el proyecto VIBORA (Morales, Aguerra, 2003) como un sistema administrador de cursos.

Con el uso de los objetos de aprendizaje podemos evitar el re-trabajo tecnológico, redescubriendo soluciones queya hayan sido cubiertas con anterioridad, garantizando construir materiales de calidad y teniendo la alternativa de poder re-usar el material ya construido. Resulta como un beneficio el aprovechar la potencialidad de las herramientas tecnológicas que permiten hacer de los objetos de aprendizaje un elemento electrónico de aprendizaje fácil de acceder y compartir.

Se percibe una necesidad de crear $L O$ dado que aumenta la versatilidad y la funcionalidad de los materiales didácticos, se presenta una mayor disponibilidad de contenidos, con un soporte más amplio y distribuido. Los principales beneficios se apreciarán en la instituciones al combinar esfuerzos y compartir resultados dado que al compartir y re-utilizar se producen ahorros de recursos que se pueden redirigir hacia más desarrollo tecnológico.

Visualizando la aplicación que ofrecen los $L O$ estos deberán ser compatibles con diversos ambientes y sistemas de administración de aprendizajes, fáciles de migrar de una plataforma a otra y fáciles de localizar, acceder, archivar y re-utilizar. Los potenciales beneficios de los $L O$ son la reutilización, interoperatividad, la durabilidad, la accesibilidad, la colaboración y la personalización. Estos requerimientos se tornan a estándares apropiados para el diseño y descripción de los objetos de aprendizaje.

La incorporación de los $L O$ en el desarrollo de la metodología propuesta en trabajos preliminares se debe a que tecnológicamente es un nuevo tipo de medio electrónico digitalizado que apoya al proceso enseñanza-aprendizaje. 
Ma. DE Lourdes Margain, Francisco Álvarez y Jaime MuñoZ

COLABORACIÓN DE LOS OBJETOS DE APRENDIZAJE EN LA GESTIÓN DEL APRENDIZAJE

Aunando los conceptos de colaboración y objetos de aprendizaje, el aspecto colaboración entre estudiantes, refiere a que cada estudiante se hará responsable tanto del aprendizaje de los otros como del suyo propio. El aspecto de la colaboración entre los maestros, referirá sobre la evaluación del aprendizaje y por último, la colaboración de los objetos sobre el rol o el papel que juega en el proceso enseñanzaaprendizaje (PEA). Por lo tanto y, desde este punto de vista, surge la siguiente interrogante: ¿Cómo es que los LO colaboran en la gestión del aprendizaje?

\section{PROBLEMÁTICA}

Existen varios momentos dentro del Proceso Enseñanza Aprendizaje (PEA) en los sistemas de gestión de aprendizaje en que la problemática de la gestión del aprendizaje se acentúa, en este trabajo se aborda en tres fases fundamentales (ver Figura 1).

Primera fase: Cuando los estudiantes se encuentran en el proceso de adquirir, generar y procesar la información proporcionada por el maestro; claro es que el aprendizaje colaborativo no se da si éste se fundamenta únicamente en repartir el trabajo entre los integrantes del equipo para posteriormente solamente unirlo, deberá existir una reflexión, discusión, análisis y toma de decisiones respecto al trabajo realizado, debe fomentarse una responsabilidad individual y una hacia el grupo.

Segunda fase: Cuando por parte del profesor existe la necesidad de incorporar en los estudiantes, nuevas destrezas ya sea de manera individual o en grupo y como consecuencia de esta incorporación de nuevo conocimiento, se requiere evaluar el aprendizaje obtenido.

Tercera fase: Cuando se generan los elementos resultantes de la colaboración ente maestros y alumnos, como ideas, procesos mentales, propuestas, recomendaciones, acuerdos, desacuerdos y mal entendidos. 


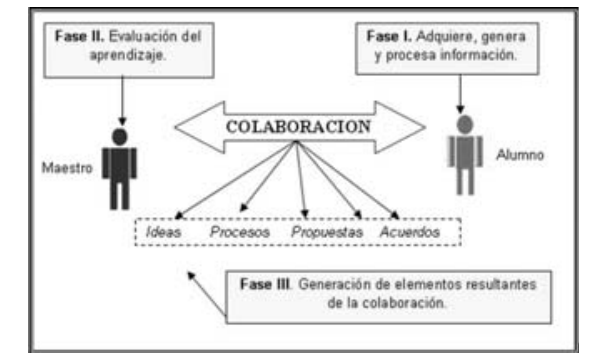

Fig. 1. Problemática de gestión del aprendizaje.

Para que se facilite la gestión del aprendizaje es necesario determinar el "know-how" de la gestión, esto es, saber plasmar el aspecto de colaboración entre estudiantes, maestros. El saber como el estudiante adquirirá, generará y procesará información, el saber como por parte del maestro puede evaluar el aprendizaje y el saber como se puede provocar que se generen los elementos resultantes de la colaboración, en resumen se requiere conocer los beneficios que trae consigo el aspecto de la colaboración y como respuesta a este "know-how" aparecen los objetos de aprendizaje planteados de una manera natural, haciendo que el mismo objeto de aprendizaje también obtenga una labor colaborativa en el proceso dentro de las fases identificadas anteriormente.

Fase I. Colaboración de los LO en la gestión del aprendizaje en la adquisición, generación y procesamiento de la información.

La colaboración de los objetos se da en el fomento de la tareas colaborativas, ya no es función únicamente del maestro enseñar, si no ahora el alumno aprende a aprender y a usar y crear sus propios recursos digitales (objetos de aprendizaje). La colaboración de los LO ayudará en la medida de lograr el objetivo de las tareas si los demás miembros del grupo también lo hacen, como se muestra en la figura 2.

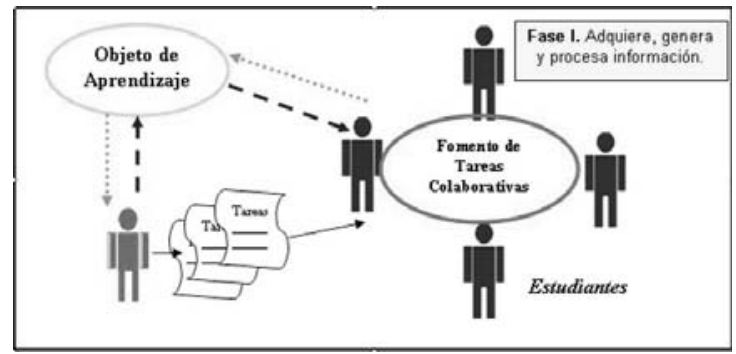

Fig. 2. Colaboración de los objetos de aprendizaje en la Fase I 
Ma. DE Lourdes Margain, Francisco Álvarez y Jaime MuñoZ

COLABORACIÓN DE LOS OBJETOS DE APRENDIZAJE EN LA GESTIÓN DEL APRENDIZAJE

En la Fase I, los objetos de aprendizaje basándonos en sus características naturales definidas por David Wiley (2000), se convierten en recursos digitales los cuales son aprovechados por los estudiantes, habilitando así la posibilidad de potenciar el proceso colaborativo en el fomento de las tareas colaborativas y definiendo en esta propuesta los tipos de colaboración generadas por los $L O$ como se muestra en la figura 3.
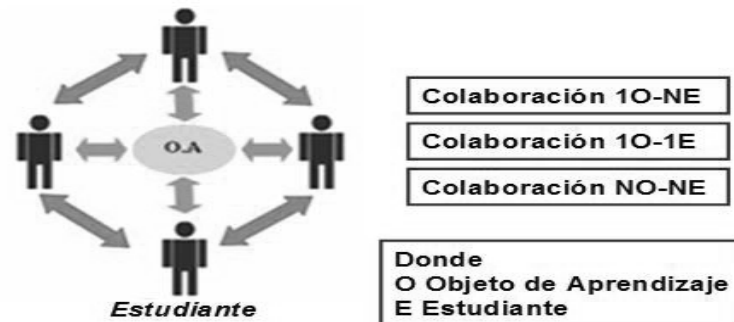

Fig. 3. Tipos de colaboración generadas por LOs Objetos de Aprendizaje

Definiremos tres tipos de colaboración generadas por los objetos de aprendizaje, la colaboración un objeto con $n$ estudiantes, donde un objeto puede ser creado, modificado y reutilizado por $n$ estudiantes. La colaboración particular de un objeto y un estudiante se da cuando un estudiante genera su propio objeto y lo comparte al resto del grupo para trabajarlo y fomenta la colaboración, y finalmente la colaboración de $n$ objetos con $n$ estudiantes donde todos generan objetos para cumplir con la misión siendo estos reutilizados por los mismos $n$ estudiantes.

Como consecuencia del trabajo de colaboración de los objetos de aprendizaje y del fomento de las tareas colaborativas se desarrollan algunas características en los aprendices como: autonomía, independencia, motivación, interactividad, solución de problemas, iniciativa, interacción, intercambio de información, manejo de conflictos, co-construcción, interpretación, negociación desarrollo de habilidades para trabajar en grupo y desarrollo de competencias, por ejemplo como las que define Cabecero (2000) competencias tecnológicas, de comunicación, animación y teóricas, entre otras.

Una vez comprendida la colaboración de los $L O$ en la adquisición, generación y procesamiento de la información a través del fomento de las tareas colaborativas, se requiere conocer la colaboración de los objetos de aprendizaje en la evaluación. 
Ma. De Lourdes Margain, Francisco Álvarez y Jaime MuÑoz

COLABORACIÓN DE LOS OBJETOS DE APRENDIZAJE EN LA GESTIÓN DEL APRENDIZAJE

\section{Fase II. Colaboración de los LO en la gestión del aprendizaje para la evaluación del aprendizaje.}

Una aportación más de los LO en la gestión del aprendizaje se da en lo referente a la evaluación: Pero, ¿Cómo es que LOs objetos de aprendizaje colaborarán en el proceso de la evaluación del aprendizaje?.

La Comisión Académica de Objetos de Aprendizaje del CUDI (2002) hace una lista detallada de los elementos que un $L O$ debería contener desde el punto de vista del diseño. Uno de estos elementos que se consideran relevantes para el diseño es lo correspondiente a la evaluación tanto del objeto como de la evaluación del aprendizaje usando $L O$. Concerniente a la evaluación del objeto, algunos autores expertos en formación de autores (Navarro, 2005) consideran que el objeto tiene que contemplar una forma de evaluar si los propósitos del objeto se verifican. Otros autores bajo otro enfoque trabajan en modelos para la evaluación del aprendizaje mediante LO. (Guardia, 2004)

En la literatura revisada, encontramos que el tema de aprendizaje colaborativo es preocupación y objeto de estudio en varios países, en investigaciones actuales en Chile, Álvarez (2004) clasifica los objetos de aprendizaje de acuerdo a su uso pedagógico en objetos de instrucción, de colaboración, de prácticas y de evaluación. Particularmente en este trabajo se retoma y se profundiza sobre los objetos de evaluación los cuales se encuentran "colaborando" entre maestros y estudiantes. Ver figura 4 .

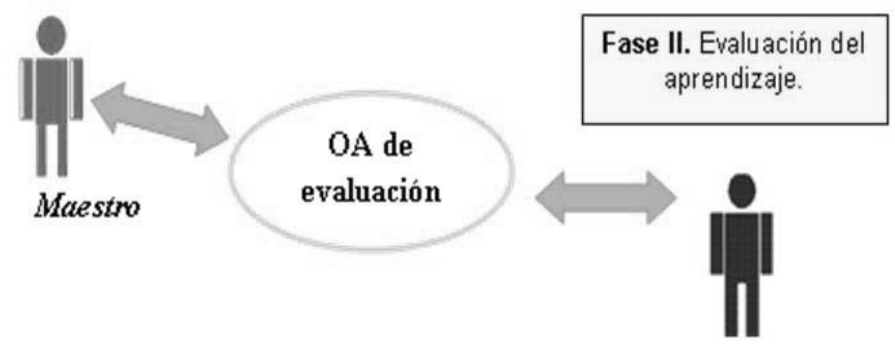

Fig. 4. Colaboración de LOs LO en la Fase II

Álvarez (2004) define los objetos de evaluación como:

"Objetos que tienen como función conocer el nivel de conocimiento que tiene un aprendiz" 
Ma. De Lourdes Margain, Francisco Álvarez y Jaime Muñoz

COLABORACIÓN DE LOS OBJETOS DE APRENDIZAJE EN LA GESTIÓN DEL APRENDIZAJE

Y plasma cuatro categorías:

- De Pre-evaluación: Son objetos destinados a medir el nivel de conocimiento que tiene un aprendiz antes de comenzar el proceso de aprendizaje.

- De Evaluación de Proficiencia: Estos objetos sirven para medir si un aprendiz ha asimilado determinados contenidos que permitan deducir una habilidad. Por ejemplo, si un aprendiz obtiene una determinada puntuación en un test, se puede considerar que ha cumplido LOs objetivos en el camino del aprendizaje y está listo para realizar una determinada tarea o asumir un determinado rol.

- De Test de Rendimiento: Estos objetos, se usan para medir la habilidad de un aprendiz en una tarea muy específica. Usualmente son aplicaciones basadas en GUI (Graphic Unit Interfaces) compuestas de varios niveles de dificultad que el aprendiz debe ir superando y al final se le entrega un resumen de su desempeño. Este tipo de objetos normalmente se usa con objetos de simulación.

- De Pre-Test de Certificación: Usados generalmente al final de un programa orientado a la certificación y son usados en dos modos: estudio y certificación. En la modalidad de estudio el objeto es diseñado para maximizar el aprendizaje entregando un listado de LOs errores, mientras que en el modo de certificación es diseñado de manera similar a un examen final.

Adicionalmente a esta categorización de los objetos, en este trabajo se propone determinar la forma de evaluación entre el evaluador y el evaluado.

Ver Tabla 1.

\begin{tabular}{|c|c|c|}
\hline Forma de Evaluación & Evaluador & Evaluado \\
\hline Directa & Maestro & Estudiante $n$ \\
\hline Colaborativa & Estudiante(s) $m$ & Estudiante $n$ \\
\hline Auto-evaluación & Estudiante $n$ & Estudiante $n$ \\
\hline
\end{tabular}

Tabla 1. Forma de evaluación de aprendizaje

- La evaluación directa: Se define como el uso de objetos de aprendizaje, siendo aplicados directamente del maestro al alumno. 
Ma. De Lourdes Margain, Francisco Álvarez y Jaime MuÑoz

COLABORACIÓN DE LOS OBJETOS DE APRENDIZAJE EN LA GESTIÓN DEL APRENDIZAJE

- La evaluación colaborativa: Es el uso de los recursos digitales con la finalidad de que la evaluación se dé entre los mismos miembros (estudiantes) de un grupo.

- La auto-evaluación: Es el uso de los objetos utilizando el recurso del autLOprendizaje, es decir es el mismo estudiante quien podrá medir su nivel de conocimiento.

Si a estas categorías de objetos de aprendizaje de evaluación ( $L O e$ ) las combinamos con la forma de evaluación, comprobamos que la colaboración de los $L O$ fortalece la gestión al tener la posibilidad de medir el nivel de conocimiento que se adquiere con el uso de los $L O$. Ver Tabla 2.

\begin{tabular}{|l|c|c|c|}
\hline \multicolumn{1}{|c|}{ Tipo de Objeto } & Directa & Colaborativa & Auto evaluación \\
\hline Pre-evaluación & $\mathrm{Si}$ & $\mathrm{Si}$ & $\mathrm{Si}$ \\
\hline Proficiencia & $\mathrm{Si}$ & $\mathrm{Si}$ & $\mathrm{Si}$ \\
\hline Test de Rendimiento & $\mathrm{Si}$ & $\mathrm{Si}$ & $\mathrm{Si}$ \\
\hline Pre-Test de Certificación & $\mathrm{Si}$ & $\mathrm{Si}$ & $\mathrm{Si}$ \\
\hline
\end{tabular}

Tabla 2. Categorías de los LO de evaluación vs. forma de evaluación

Fortalecida la colaboración de los $L O$ de evaluación, a través de la combinación de las categorías de los LOe con la forma de evaluación, por último y como alcance de este artículo se hace conocer la contribución de los objetos de aprendizaje en la generación de los elementos resultantes de la colaboración ente alumnos y maestros.

Fase III. Colaboración de los LO en la gestión del aprendizaje en los elementos resultantes de la colaboración.

En investigaciones preliminares de los mismos autores de este trabajo (Margain, 2005), se propone para la gestión del aprendizaje modelar el grado de colaboración, con el interés de aprender, entre aprendices y maestros dado que en el momento que los actores (estudiantes-maestros) colaboran, se generan algunos elementos como ideas, procesos mentales, propuestas, acuerdos, desacuerdos, mal entendidos, etc. Estos elementos requieren ser captados y una propuesta es utilizando mapas conceptuales por medio de ambientes informáticos colaborativos con el fin de gestionarlos. 
En este artículo, estos elementos se tratan desde la perspectiva de el saber como con la incorporación y uso de los $L O$, se puede provocar que se generen estos elementos.

Para cerrar el ciclo del Proceso Enseñanza Aprendizaje y además facilitar la gestión del aprendizaje, se requiere que posteriormente a obtener el resultado de la evaluación, exista una retroalimentación del maestro al alumno, en este sentido el maestro puede ocupar los mismos objetos de aprendizaje que fueron creados y/o modificados por los estudiantes, dado que el objeto puede ser transformado o actualizado para indicar la precisión del aprendizaje esperado.

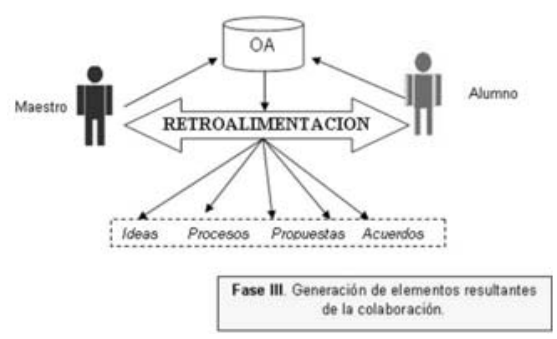

Fig. 5. Colaboración de los LO en la Fase III

De esta manera, en esta fase (ver figura 5) la colaboración de los objetos es identificada como retroalimentación donde el alumno genera nuevas ideas, procesos mentales, propuestas, acuerdos, desacuerdos, mal entendido(s), etc.

\section{Conclusiones y Trabajo Futuro}

Consolidada la contribución de los $L O$ en la generación de estos elementos a través de la retroalimentación de la evaluación (fase II), se identifica la necesidad de conocer dos aspectos fundamentales resultantes de la colaboración de los $L O$ en la gestión del aprendizaje: Los métricos y el impacto; por un lado, se debe medir el nivel de conocimiento que se adquiere con el uso de los $L O$ y por otro lado conocer el impacto que tiene el uso de los mismos en el aprendizaje. Estos puntos serán tratados posteriormente.

En conclusión, como resultado de la colaboración de los $L O$ se clarifica el "Know How" en la gestión del aprendizaje. Se obtiene la certeza de que la mayor ventaja de incorporar $L O$ y utilizarlos se deriva de la verdadera interacción que surge entre los actores (estudiantes-maestros-objetos). 
Ma. DE Lourdes Margain, Francisco Álvarez y Jaime Muñoz

COLABORACIÓN DE LOS OBJETOS DE APRENDIZAJE EN LA GESTIÓN DEL APRENDIZAJE

\section{REFERENCIAS BIBLIOGRÁFICAS}

Ausubel, D. P, J. D. Novak, \& H. Hanesian (1978). Educational Psychology: A Cognitive View (2a edición). New York: Holt, Rinehart \& Winston. Reimpreso, 1986. New York: Warbel \& Peck.

Aguilar, L y otros. (2003). Observatorio Ciudadano de la Educación. Disponible en: http://www.observatorio.org/ comunicados/comun108.html. [consulta 22 de febrero 2006].

Álvarez, L. (2004). Conjuntos Difusos de Objetos de Aprendizaje. Instituto de Informática. Universidad Austral de Chile. Disponible en: http://www.inf. uach.cl/lalvarez/documentos.htm

Bermejo, S., Treviño, M. E., (2003): “Objetos de aprendizaje personalizados”, XI Congreso Universitario de Innovación Educativa en las Enseñanzas Técnicas.

Cabecero, J. (200). La formación virtual: principios, bases y preocupaciones. Universidad de Sevilla.

Guàrdia, L. \& Sangrà, A. (2004). Diseño instruccional y objetos de aprendizaje: hacia un modelo para el diseño de actividades de evaluación del aprendizaje on-line. Estudios de Psicología y Ciencias de la Educación, Universitat Oberta de Catalunya, España.

Margain, L., Álvarez, F., Muñoz, J. (2005). Método de Aprendizaje basado en Mapas Conceptuales y Objetos de Aprendizaje "MACOBA". Universidad Politécnica de Aguascalientes, Universidad Autónoma de Aguascalientes. Encuentro Internacional de Educación a Distancia, ENCo5. Guadalajara. México.

Morales, Aguerra (2003). El proyecto VIBORA de capacitación en web con objetos de aprendizaje. ENC 03

Navarro, J. (2005). Objetos de Aprendizaje, Formación de autores con el modelo de redes de objetos. Universidad de Guadalajara. México, 2005.

Veja, G., Rojo, B. (1999). Educación a Distancia y Aprendizaje Colaborativo, Centro Educación a Distancia, Ponencia presentada en las III Jornadas de Educación a Distancia - Mercosur '99" organizadas por el CREAD en la Universidad de Los Lagos, Osorno Chile.

Vizcaíno, A., Olivas, J., Prieto, M., (2001). Modelos del estudiante en entornos de aprendizaje colaborativo, Escuela Superior de Informática. Universidad de Castilla-La Mancha. España.

Wiley, D.,(2000). Learning object design and sequencing theory, Department of InstructionalPsychologyandTechnology, Brigham Young University.

Zapata, M. (2003). Evaluación de un Sistema de Gestión del Aprendizaje. Disponible en: http://www.um.es/ead/aula/calidad/ plataformas/eval SGA beta 1.pdf 
Ma. DE Lourdes Margain, Francisco Álvarez y Jaime Muñoz

COLABORACIÓN DE LOS OBJETOS DE APRENDIZAJE EN LA GESTIÓN DEL APRENDIZAJE

\section{PALABRAS CLAVE}

Aprendizaje colaborativo, Objetos de Aprendizaje, Gestión del aprendizaje, Aprendizaje en línea.

\section{KEY WORDS}

Collaborative Learning, Learning Objects, Learning Management, e-learning.

\section{PERFIL ACADÉMICO DE LA AUTORA}

Ma. de Lourdes Margain Fuentes, realiza sus estudios de Postgrado en la UAA y obtiene el grado de Maestra en Informática y Tecnologías Computacionales en el año 2004. Tesis: "Diseño y Evaluación de un Modelo Conceptual para la Interpretación de Evaluaciones de los Procesos de Ingeniería del Modelo Integrado de Madurez de Capacidades” en la línea de Ingeniería de Software. Actualmente cursa el Doctorado en Ciencias Computacionales motivada por la Educación a Distancia para profundizar en Objetos de Aprendizaje.

Dirección postal: $\quad$ Av. Prolongación Mahatma Gandhi Km. 2

Col. San Francisco del Arenal CP: 20180

Aguascalientes, Ags. (México)

Universidad Politécnica de Aguascalientes

lourdes.margain@upa.edu.mx

fjalvar@correo.uaa.mx,

jmunozar@correo.uaa.mx

Fecha de entrega: 06.10.06

Fecha de aceptación: 10.12.06 